\title{
Correspondence between general practitioner- reported medication use and timing of prescription dispensation
}

This article was published in the following Dove Press journal:

Clinical Epidemiology

4 January 2012

Number of times this article has been viewed

\author{
Sigrun Alba Johannesdottir \\ Merete Lund Mægbæk \\ Jens Georg Hansen \\ Timothy L Lash \\ Lars Pedersen \\ Vera Ehrenstein
}

Department of Clinical Epidemiology, Institute of Clinical Medicine, Aarhus University Hospital, Aarhus, Denmark
Correspondence: Sigrun Alba Johannesdottir

Department of Clinical Epidemiology,

Aarhus University Hospital,

Olof Palmes Allé 43-45,

8200 Aarhus N, Denmark

Tel +45 23695947

$\mathrm{Fax}+45$ 87I 672I5

Email saj@dce.au.dk

\begin{abstract}
Epidemiologic studies often rely on drug dispensation records to measure medication intake. We aimed to estimate correspondence between general practitioner (GP)-reported treatment and timing of prescription dispensation. From seven GPs in northern Denmark, we obtained 317 prescription records for 286 patients treated with ten commonly prescribed medication types for chronic diseases. We linked the GP-reported information to the regional prescription database to retrieve patients' prescription records both prospectively and retrospectively in relation to the GP-reported date of treatment (index date, August 20, 2008 for all patients). We computed overall and medication-specific correspondence between GP-reported treatment and the timing of dispensation. We computed correspondence based on both exact medication and therapeutic subgroup agreement. The correspondence for dispensation within \pm 90 days of GP-reported treatment was $0.81(95 \%$ confidence interval $=0.76-0.85)$ with variation by medication type, ranging from 0.55 for ACE-inhibitors to 1.00 for oral glucose-lowering agents. The correspondence was greater when analyzed within therapeutic groups than when analyzed for exact medications within these groups.
\end{abstract}

Keywords: pharmacoepidemiology, predictive value, drug prescriptions, primary health care

\section{Introduction}

Pharmacoepidemiology studies often rely on drug dispensation records in prescription registries to measure medication intake. ${ }^{1,2}$ Despite many advantages (eg, large size and unselected sample), ${ }^{3}$ registry data merely approximate the true medication intake, reflecting neither its fact nor the timing. ${ }^{1,4}$ This limitation necessitates an assumption of good correspondence between dispensation record and drug intake. ${ }^{4}$ If untenable, such assumption may introduce misclassification of the true drug intake, potentially producing misleading results. ${ }^{4}$

Nevertheless, dispensation data are considered superior to most other measures of drug intake, and validation studies commonly use them as a gold standard to assess data quality on medication intake reported by patients ${ }^{5,6}$ or their proxies. ${ }^{7}$ However, little is known about the correspondence between the actual treatment and the timing of dispensation. We therefore examined the correspondence between general practitioner (GP)-reported treatment with prescription medication and the timing of dispensation, using a prescription registry in Denmark.

\section{Materials and methods}

Denmark is a welfare state, with universal tax-funded health care. ${ }^{8}$ GPs are gatekeepers to patients' health care utilization, by providing referrals to hospitals and specialists and by 
prescribing medicine. ${ }^{8}$ The primary care sector is responsible for most of the outpatient prescribing in Denmark, accounting for $96 \%$ of the total volume of sold medicinal products in 2009. ${ }^{9}$ Prescriptions dispensed in outpatient pharmacies are recorded in prescription registries, with information on date of dispensation and Anatomical Therapeutic Chemical (ATC) classification code of the medicine. ${ }^{10}$

We obtained data on treatment with reimbursed prescription medications from seven general practices in the North Jutland region. On a randomly selected calendar date (August 20, 2008, the index date), each GP was asked to search his or her electronic records to randomly identify up to five patients for each of the ten agents on a pre-determined list of prescription medications, commonly used to treat chronic diseases: ${ }^{9}$ proton pump inhibitors; oral glucose-lowering drugs; acetylsalicylic acid; diuretics; ACE inhibitors; statins; systemic glucocorticoids; nonsteroidal anti-inflammatory drugs (NSAIDs); bisphosphonates; and adrenergic inhalants. Each reported medication corresponded to one prescription record, so that patients using two agents of interest contributed two prescription records to the analysis. The GP report of medication intake was considered to represent the actual treatment with a given medication on the index date. Associated ATC codes are presented in the Tables. Via the unique personal identifier, used in all official records in Denmark, ${ }^{11}$ we linked the GP-reported treatment data to the patients' records in the Aarhus University Prescription Database, which is a regularly-updated research copy of regional prescription records, maintained at our academic department. ${ }^{10}$
We estimated the correspondence between GP report and dispensation record as the proportion of GP-provided prescription records with a corresponding record in the prescription database for the same patient. We computed correspondence based on (1) full ATC code agreement, that is, correspondence of the entire ATC code, and (2) therapeutic subgroup agreement, defined as correspondence of the first three ATC code positions (eg, A02). We estimated the cumulative correspondence for 30, 90, 180, 270, and 365 days before the index date; 30, 90, and 180 days after the index date; \pm 30 days; and \pm 90 days. Furthermore, we computed the median and interquartile range of retrospective and prospective time intervals between index date and dispensation date. All analyses were performed overall and for categories of medications listed above. Finally, we stratified the results by patient age and gender.

During the month leading up to the index date (July 20-August 19, 2008), using open-ended questions (presented in the Appendix), the study nurse interviewed all patients identified from one GP practice; this was done to estimate patient-reported adherence to the prescribed treatment.

\section{Results}

Each GP supplied 39 to 50 prescription records, for a total of 317 prescription records written for 286 patients. Median age of the patients was 63 years (range, 12-93) and 51\% were men.

Overall, correspondence based on full ATC code agreement was $0.46(95 \%$ confidence interval $[\mathrm{CI}]=0.41-0.52)$ for dispensation within 30 days before the index date; $0.36(95 \% \mathrm{CI}=0.30-0.41)$ for 30 days after the index

Table I Correspondence and 95\% confidence intervals of general practitioner-reported treatment and timing of prescription dispensation according to time relative to index date ${ }^{\mathrm{a}}$ : full agreement and therapeutic group correspondence ${ }^{\mathrm{b}}$

\begin{tabular}{|c|c|c|c|c|c|}
\hline \multirow[t]{2}{*}{ Correspondence of treatment } & \multicolumn{5}{|c|}{ Days relative to the index date } \\
\hline & $\mathbf{N}$ & \pm 30 & \pm 90 & -365 & -270 \\
\hline Full ATC agreement & & $0.66(0.60-0.7 I)$ & $0.8 \mathrm{I}(0.76-0.85)$ & $0.84(0.79-0.88)$ & $0.83(0.78-0.87)$ \\
\hline Therapeutic group agreement & & $0.78(0.73-0.82)$ & $0.95(0.92-0.97)$ & $0.97(0.94-0.98)$ & $0.96(0.93-0.98)$ \\
\hline \multicolumn{6}{|c|}{ Correspondence of treatment (full ATC correspondence) by therapeutic subgroup } \\
\hline A02BC proton pump inhibitors & 34 & $0.71(0.53-0.85)$ & $0.82(0.65-0.93)$ & $0.82(0.65-0.93)$ & $0.82(0.65-0.93)$ \\
\hline AIOB oral glucose-lowering drugs & 34 & $0.94(0.80-0.99)$ & 1.00 & 1.00 & 1.00 \\
\hline B0IAC06 acetylsalicylic acid & 30 & $0.67(0.47-0.83)$ & $0.93(0.78-0.99)$ & $0.97(0.83-1.00)$ & $0.97(0.83-1.00)$ \\
\hline C03 diuretics & 33 & $0.64(0.45-0.80)$ & $0.88(0.72-0.97)$ & $0.88(0.72-0.97)$ & $0.79(0.6 I-0.9 I)$ \\
\hline C09A ACE inhibitors, plain & 31 & $0.45(0.27-0.64)$ & $0.55(0.36-0.73)$ & $0.65(0.45-0.81)$ & $0.61(0.42-0.78)$ \\
\hline CIOAA statins & 33 & $0.73(0.54-0.87)$ & $0.97(0.84-1.00)$ & $0.97(0.84-1.00)$ & $0.97(0.84-1.00)$ \\
\hline H02AB systemic glucocorticoids & 25 & $0.40(0.21-0.6 I)$ & $0.56(0.35-0.76)$ & $0.64(0.43-0.82)$ & $0.64(0.43-0.82)$ \\
\hline MOIA nonsteroidal anti-inflammatory drugs & 35 & $0.86(0.70-0.95)$ & $0.86(0.70-0.95)$ & $0.86(0.70-0.95)$ & $0.86(0.70-0.95)$ \\
\hline M05B bisphosphonates & 29 & $0.45(0.26-0.64)$ & $0.76(0.56-0.90)$ & $0.83(0.64-0.94)$ & $0.83(0.64-0.94)$ \\
\hline R03A adrenergics, inhalants & 33 & $0.6 \mathrm{I}(0.42-0.77)$ & $0.70(0.5 \mathrm{I}-0.84)$ & $0.73(0.54-0.87)$ & $0.73(0.54-0.87)$ \\
\hline
\end{tabular}


date; $0.66(95 \% \mathrm{CI}=0.60-0.71)$ for \pm 30 days, and 0.81 (95\% CI $=0.76-0.85)$ for \pm 90 days (Table 1$)$. In the period preceding the index date, correspondence rose to 0.81 at 180-days and increased only slightly from then until one year before the index date. Correspondence varied by medication and achieved 1.00 (100\% agreement) only for oral glucoselowering agents, within \pm 90 days relative to the index date and within 180 days before the index date. For remaining categories, the maximum correspondence achieved during the observation time ranged from 0.64 for systemic glucocorticoids to 0.97 for acetylsalicylic acid and statins within 180 to 365 days before the index date. Within \pm 90 days, correspondence ranged from 0.55 for ACE-inhibitors to 1.00 for oral glucose-lowering agents.

The correspondence was greater when computed based on therapeutic subgroup of the ATC code (data not shown). Under this definition, within \pm 90 days of the index date correspondence reached a value of 1.00 for proton pump inhibitors, ACEinhibitors, statins, and systemic glucocorticoids, and exceeded 0.90 for all remaining medications, except for bisphosphonates (correspondence $=0.83$ ).

Median time between the index date and dispensation retrospectively and prospectively was 24 days and 33 days, respectively (Table 2). Median time ranged from 12 days for NSAIDs to 55 days for diuretics retrospectively and from 7.5 days for NSAIDs to 72 days for systemic glucocorticoids prospectively.

The retrospective and prospective time interval between index date and dispensation date was shorter for women than for men, with variations according to medication category (Table A1). There was no material difference in correspondence according to patient age.

Of the 40 patients who were interviewed about their drug intake, $78.0 \%(95 \% \mathrm{CI}=62.0 \%-89.2 \%)$ confirmed taking prescription medication reported by their GP.

\section{Discussion}

To our knowledge, the present study is the first to examine the time dependence of correspondence between GP-reported treatment and dispensation for a wide range of medications. Dispensations registered in the prescription database before the GP report corresponded better with that report than prescriptions registered subsequently, for the time window of the same size. The correspondence was greater for therapeutic groups than for specific drugs within these groups, suggesting that physicians sometimes misreported the specific drug prescribed to their patients, but less often misreported the intended therapeutic target. Alternatively, another physician may have prescribed a different medication within the same therapeutic group (eg, during a hospital admission).

The correspondence of GP-reported treatment varied according to the type of medication. Not surprisingly, analgesics (NSAIDs) and medications that prevent lifethreatening conditions, such as oral glucose-lowering agents, showed shorter median time to dispensation and higher correspondence within shorter time span, than other medications. Absence of full correspondence between GP-reported treatments and dispensation records in the prescription database may be explained by non-adherence, discontinuation elected by the patient, or use of extra-regional

\begin{tabular}{llllll}
\hline-180 & -90 & -30 & +30 & +90 & +180 \\
\hline $0.81(0.76-0.85)$ & $0.73(0.68-0.78)$ & $0.46(0.4 I-0.52)$ & $0.36(0.30-0.4 I)$ & $0.63(0.57-0.68)$ & $0.73(0.68-0.78)$ \\
$0.93(0.90-0.96)$ & $0.85(0.8 I-0.89)$ & $0.55(0.49-0.60)$ & $0.46(0.4 I-0.52)$ & $0.77(0.72-0.8 I)$ & $0.88(0.84-0.9 I)$ \\
& & & & & \\
$0.82(0.65-0.93)$ & $0.7 I(0.53-0.85)$ & $0.4 I(0.25-0.59)$ & $0.44(0.27-0.62)$ & $0.68(0.49-0.83)$ & $0.7 I(0.53-0.85)$ \\
1.00 & $0.97(0.85-1.00)$ & $0.74(0.56-0.87)$ & $0.56(0.38-0.73)$ & $0.9 I(0.76-0.98)$ & $0.94(0.80-0.99)$ \\
$0.93(0.78-0.99)$ & $0.83(0.65-0.94)$ & $0.43(0.25-0.63)$ & $0.30(0.15-0.49)$ & $0.63(0.44-0.80)$ & $0.87(0.69-0.96)$ \\
$0.73(0.54-0.87)$ & $0.73(0.54-0.87)$ & $0.27(0.13-0.46)$ & $0.39(0.23-0.58)$ & $0.73(0.54-0.87)$ & $0.85(0.68-0.95)$ \\
$0.61(0.42-0.78)$ & $0.52(0.33-0.70)$ & $0.39(0.22-0.58)$ & $0.10(0.02-0.26)$ & $0.35(0.19-0.55)$ & $0.55(0.36-0.73)$ \\
$0.97(0.84-1.00)$ & $0.91(0.76-0.98)$ & $0.58(0.39-0.75)$ & $0.30(0.16-0.49)$ & $0.64(0.45-0.80)$ & $0.85(0.68-0.95)$ \\
$0.64(0.43-0.82)$ & $0.52(0.31-0.72)$ & $0.32(0.15-0.54)$ & $0.20(0.07-0.4 I)$ & $0.36(0.18-0.57)$ & $0.48(0.28-0.69)$ \\
$0.83(0.66-0.93)$ & $0.66(0.48-0.81)$ & $0.54(0.37-0.7 I)$ & $0.60(0.42-0.76)$ & $0.66(0.48-0.8 I)$ & $0.69(0.5 I-0.83)$ \\
$0.79(0.60-0.92)$ & $0.76(0.56-0.90)$ & $0.45(0.26-0.64)$ & $0.14(0.04-0.32)$ & $0.69(0.49-0.85)$ & $0.69(0.49-0.85)$ \\
$0.73(0.54-0.87)$ & $0.64(0.45-0.80)$ & $0.45(0.28-0.64)$ & $0.42(0.25-0.61)$ & $0.55(0.36-0.72)$ & $0.6 I(0.42-0.77)$ \\
\hline
\end{tabular}

Notes: ${ }^{\mathrm{T}}$ The index date is a randomly selected calendar date (August 20,2008) on which the general practitioners searched their electronic records for patients treated with the listed agents. 'Full correspondence is defined as full correspondence of the drug's Anatomical Therapeutic Chemical (ATC) code; therapeutic group agreement is defined as correspondence of at least first three positions of the ATC code.

Abbreviation: ACE, angiotensin converting enzyme. 
Table 2 Median and interquartile range of time (days) elapsed until dispensation of medication relative to the index date ${ }^{a}$

\begin{tabular}{|c|c|c|c|c|c|}
\hline \multirow[t]{2}{*}{ Therapeutic subgroup } & \multirow[t]{2}{*}{ ATC-code ${ }^{b}$} & \multicolumn{2}{|c|}{ Retrospective } & \multicolumn{2}{|c|}{ Prospective } \\
\hline & & Median & Interquartile range & Median & Interquartile range \\
\hline Proton pump inhibitors & $\mathrm{A} 02 \mathrm{BC}$ & 35 & $10-75$ & 20 & $6-43$ \\
\hline Oral glucose-lowering drugs & $\mathrm{AIOB}$ & 17 & $12-39$ & 25 & $13-35$ \\
\hline Acetylsalicylic acid & B0IAC06 & 48 & $7-77$ & 55 & $22-93$ \\
\hline Diuretics & $\mathrm{CO3}$ & 55 & $13-82$ & 42 & $15-89$ \\
\hline ACE inhibitors, plain & C09A & 19 & $11-69$ & 54 & $34-97$ \\
\hline Statins & CIOAA & 19 & $7-53$ & 64 & $11-92$ \\
\hline Systemic glucocorticoids & $H 02 A B$ & 36 & $7-65$ & 72 & $15-84$ \\
\hline Nonsteroidal anti-inflammatory drugs & MOIA & 12 & $4-82$ & 7.5 & $0-20$ \\
\hline Bisphosphonates & M05BA & 29 & $13-58$ & 52 & $38-67$ \\
\hline Adrenergics, inhalants & R03A & 18 & $7-53$ & 27 & $16-39$ \\
\hline Overall & All above & 24 & $8-63$ & 33 & $13-72$ \\
\hline
\end{tabular}

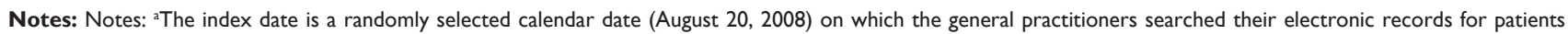
treated with the listed agents. ${ }^{b}$ Anatomical Therapeutic Chemical (ATC) classification code.

Abbreviation: $\mathrm{ACE}$, angiotensin converting enzyme.

or hospital-based pharmacies. Furthermore, low short-term correspondence may result from a certain proportion of patients having a supply remaining from their last refill, close in time to the index date.

For the medications under study, correspondence between patients' self-report in the month before the index date was similar to the \pm 90 -day agreement. Lack of full correspondence between GP and patient report may be related not only to non-adherence, but also to recall bias and the usual variation in medication supply until the next refill on the date of the interview.

Studies similar to the present one are uncommon. In New Zealand, with health care and reimbursement schemes similar to those in Denmark, correspondence between dispensation and electronic prescribing records from a general practice was 0.97 for simvastatin; 0.98 for metoprolol; 0.95 for bendrofluazide; 0.97 for cilazapril; 1.00 for felodipine; and 0.98 for metformin. With the exception of cilazapril, these results are similar to our \pm 90 day estimates for the corresponding therapeutic subgroups (we did not include felodipine and metoprolol). ${ }^{12}$ A Dutch study found only $65 \%$ agreement between prescription and dispensation records, possibly attributable to imperfect individual linkage. ${ }^{13}$

It is reasonable to assume that correspondence, measured in this study, correlates well with adherence. The correspondence between patient and GP report as well as between GP report and dispensation in our study indicate satisfactory overall levels of adherence $(>80 \%)$ within 3 months of the reported true treatment instance. ${ }^{12,14}$ Most medications examined in this study have a 90-day refill cycle in Denmark. If the refill period was 30 days, the overall \pm 30 day correspondence of $78 \%$ likewise indicates reasonable adherence. In Sweden, refill adherence was $57 \%$ overall, with variation by medication type and prescriber type (higher for GPs than for specialists).${ }^{14}$ Contrary to that study, however, prescription issue date in our study was unknown.

Our study has several limitations. First, the sample size was relatively small, limiting our ability to obtain meaningful subgroup analysis. Second, despite the fact that we selected patients randomly, we cannot rule out selection bias. Third, because GP records in Denmark allow no search for medication non-users, we were unable to compute negative predictive values of the treatment absence against the dispensation record. Fourth, the estimates of correspondence provided here are applicable to outpatient prescriptions for reimbursed medications taken regularly. Correspondence may be lower for over-the-counter or non-reimbursed medications or for medications prescribed for acute or intermittent conditions such as those with instructions to take 'as needed'.${ }^{15}$ Finally, both the GP-reported treatment and the dispensation records are approximations to the actual intake and its timing, since it is possible that patients filled prescriptions but never took the drug.

\section{Conclusion}

The correspondence between GP-reported treatment with prescription medicine and dispensation record differs by medication type. Correspondence is greater for therapeutic group than for specific drugs within therapeutic groups, possibly reflecting situations in which physicians misreported the specific prescription, but accurately reported the therapeutic target.

\section{Acknowledgments}

We thank the seven GPs, Annemarie Nielsen, Bjarne Uhrenholt, Dan Hafstrøm, Jens Georg Hansen, Keld Gammelby Jensen, 
Klaus Borch, and Søren Sillesen, for their participation in this study. We thank Birgit Toft Sørensen for collecting data from the GPs and for conducting patient interviews. The study received financial support from the Clinical Epidemiological Research Foundation.

\section{Disclosure}

The authors report no conflicts of interest in this work.

\section{References}

1. Schneeweiss S, Avorn J. A review of uses of health care utilization databases for epidemiologic research on therapeutics. J Clin Epidemiol. 2005;58(4):323-337.

2. Ehrenstein V, Sørensen HT, Bakketeig LS, Pedersen L. Medical databases in studies of drug teratogenicity: methodological issues. Clin Epidemiol. 2010;2:37-43.

3. Sørensen HT, Baron JA. Registries and medical databases. In: Olsen J, Saracci R, Trichopoulos D, editors. Teaching Epidemiology: A Guide for Teachers in Epidemiology, Public Health and Clinical Medicine. 3rd ed. Oxford: Oxford University Press; 2009:455-466.

4. Nielsen LH, Lokkegaard E, Andreasen AH, Keiding N. Using prescription registries to define continuous drug use: how to fill gaps between prescriptions. Pharmacoepidemiol Drug Saf. 2008;17(4):384-388.

5. Lau HS, de Boer A, Beuning KS, Porsius A. Validation of pharmacy records in drug exposure assessment. J Clin Epidemiol. 1997;50(5):619-625.

6. Løkkegaard EL, Johnsen SP, Heitmann BL, et al. The validity of self-reported use of hormone replacement therapy among Danish nurses. Acta Obstet Gynecol Scand. 2004;83(5):476-481.
7. Wogelius P, Poulsen S, Sørensen HT. Validity of parental-reported questionnaire data on Danish children's use of asthma-drugs: a comparison with a population-based prescription database. Eur J Epidemiol. 2005;20(1):17-22.

8. Ministry of Interior and Health. Health Care in Denmark. Available from: http://www.sum.dk/Aktuelt/Publikationer/Publikationer/ UK_Healthcare_in_DK.aspx. Accessed September 20, 2011.

9. Danish Medicines Agency, total sales of medicinal products 2005-2009. Available from: http://laegemiddelstyrelsen.dk/ /media/1DE4CE32C6 DB4DBCBF7EF002B646980E.ashx. Accessed September 20, 2011.

10. Ehrenstein V, Antonsen S, Pedersen L. Existing data sources for clinical epidemiology: Aarhus University Prescription Database. Clin Epidemiol. 2010;2:273-279.

11. Pedersen CB. The Danish civil registration system. Scand J Public Health. 2011;39(Suppl 7):22-25.

12. Mabotuwana T, Warren J, Harrison J, Kenealy T. What can primary care prescribing data tell us about individual adherence to longterm medication? Comparison to pharmacy dispensing data Pharmacoepidemiol Drug Saf. 2009;18(10):956-964.

13. Florentinus SR, Souverein PC, Griens FA, Groenewegen PP, Leufkens HG, Heerdink ER. Linking community pharmacy dispensing data to prescribing data of general practitioners. BMC Med Inform Decis Mak. 2006;6:18.

14. Andersson K, Melander A, Svensson C, Lind O, Nilsson JL. Repeat prescriptions: refill adherence in relation to patient and prescriber characteristics, reimbursement level and type of medication. Eur J Public Health. 2005;15(6):621-626.

15. Grymonpre R, Cheang M, Fraser M, Metge C, Sitar DS. Validity of a prescription claims database to estimate medication adherence in older persons. Medical Care. 2006;44(5):471-477. 


\section{Appendix}

\section{Questionnaire used by the study nurse}

The questions regard your use of medicine, both on prescription and over-the counter.

1. Have you used any drugs within the previous week?

No Proceed to next question.

Yes What was the name(s) of the drug(s)?

Why did you take the drug?

Do you remember the dose?

2. If you answered no to the previous question:

Have you used any drugs within the previous month?

No Proceed to next question.

Yes What was the name(s) of the drug(s)?
Why did you take the drug?

Do you remember the dose?

3. Have you used any drugs within the previous three months?

No Proceed to next question.

Yes What was the name(s) of the drug(s)?

Why did you take the drug?

Do you remember the dose?

Thank you for participating.

Table AI Median and interquartile range of time (days) elapsed until dispensation of medication relative to the index date ${ }^{\mathrm{a}}$, by gender

\begin{tabular}{|c|c|c|c|c|c|}
\hline \multirow[t]{2}{*}{ Therapeutic subgroup } & \multirow[t]{2}{*}{ ATC-code ${ }^{b}$} & \multicolumn{2}{|c|}{ Retrospective } & \multicolumn{2}{|l|}{ Prospective } \\
\hline & & Women & Men & Women & Men \\
\hline Proton pump inhibitors & A02BC & $22(12-85)$ & $52(8.0-7 I)$ & $17(7.0-4 \mid)$ & $35(1.0-74)$ \\
\hline Oral glucose-lowering drugs & $\mathrm{AIOB}$ & $18(13-46)$ & $16(7.0-39)$ & $20(13-33)$ & $30(13-35)$ \\
\hline Acetylsalicylic acid & B0IAC06 & II (4.0-48) & $54.5(7.0-79)$ & $75(2.0-78)$ & $51(26-103)$ \\
\hline Diuretics & $\mathrm{C} 03$ & $5 \mathrm{I}(7.0-78)$ & $63(13-86)$ & $28(13-76)$ & $54(19-90)$ \\
\hline ACE inhibitors, plain & C09A & $9(5.0-15)$ & $25(12-148)$ & 91 (54-99) & 39 (27-89) \\
\hline Statins & CIOAA & $12(6.0-28)$ & $46(9.0-55)$ & $86(15-100)$ & $57(6.0-64)$ \\
\hline Systemic glucocorticoids & $\mathrm{H} 02 \mathrm{AB}$ & $54(9.0-61)$ & $30(5.0-123)$ & $48(15-76)$ & $72(14-92)$ \\
\hline Nonsteroidal anti-inflammatory drugs & MOIA & $43(0.0-93)$ & $9(5.0-26)$ & $7.5(0.0-19)$ & $7.5(0.0-26)$ \\
\hline Bisphosphonates & M05BA & $26(13-55)$ & $50(50-60)$ & $53(28-68)$ & $50(40-58)$ \\
\hline Adrenergics, inhalants & R03A & $23(6.0-55)$ & $16(8.0-34)$ & $24(18-35)$ & $28(9.0-45)$ \\
\hline Overall & All above & $21(7.5-62)$ & $28(8.0-7 I)$ & $26(9.0-68)$ & $36(15-72)$ \\
\hline
\end{tabular}

Notes: a The index date is a randomly selected calendar date (August 20, 2008) on which the general practitioners searched their electronic records for patients treated with the listed agents. ${ }^{b}$ Anatomical Therapeutic Chemical (ATC) classification code

Abbreviations: ACE, angiotensin converting enzyme.

Clinical Epidemiology

\section{Publish your work in this journal}

Clinical Epidemiology is an international, peer-reviewed, open access journal focusing on disease and drug epidemiology, identification of risk factors and screening procedures to develop optimal preventative initiatives and programs. Specific topics include: diagnosis, prognosis, treatment, screening, prevention, risk factor modification, systematic

\section{Dovepress}

reviews, risk \& safety of medical interventions, epidemiology \& biostatical methods, evaluation of guidelines, translational medicine, health policies \& economic evaluations. The manuscript management system is completely online and includes a very quick and fair peer-review system, which is all easy to use. 